\title{
Hydrogenated amorphous silicon deposited by high pressure sputtering for HIT solar cells
}

\author{
R. Garcia-Hernansanz*, E. Garcia-Hemme, D. Pastor, \\ A. del Prado, I. Martil, G. Gonzalez-Diaz \\ Dpto. Física Aplicada III, \\ Universidad Complutense de Madrid \\ Madrid, Spain \\ Telf. 913944434, e-mail: * rodrigo.garcia.hernansanz@,fis.ucm.es
}

J. Olea, D. Pastor

Instituto de Energía Solar

E.T.S.I. Telecomunicación, Universidad Politécnica de Madrid Madrid, Spain

\section{ABSTRACT}

\begin{abstract}
Hydrogenated amorphous silicon thin films were deposited using a high pressure sputtering (HPS) system. In this work, we have studied the composition and optical properties of the films (band-gap, absorption coefficient), and their dependence with the deposition parameters. For films deposited at high pressure (1 mbar), composition measurements show a critical dependence of the purity of the films with the RF power. Films manufactured with RF-power above $80 \mathrm{~W}$ exhibit good properties for future application, similar to the films deposited by CVD (Chemical Vapor Deposition) for hydrogenated amorphous silicon.
\end{abstract}

Keywords-hydrogenated amorphous silicon; intermediate band; high pressure sputtering; HIT cell; composition; absorption bands.

\section{INTRODUCTION}

Industry and science have performed a great economic and human effort to increase the conversion efficiency of solar cells. The HIT solar cell (heterojunction with intrinsic thinlayer) is a good exponent of that, and is one of the most investigated cell structure, due to its low manufacturing cost and its high efficiency [1]. This device consists of a heterojunction of a hydrogenated amorphous silicon (a-Si:H) film with a crystalline silicon (c-Si) substrate. Between both layers a very thin film $(\sim 5-20 \mathrm{~nm})$ of intrinsic amorphous silicon is deposited, whose principal function is to passivate the junction to improve the cell efficiency. We focus on HIT cells due to the low manufacturing temperatures for cell processes, which are below $300^{\circ} \mathrm{C}$ [2]. On the other hand, one of its challenges is to found an alternative to CVD (chemical vapor deposition) for the a-Si:H deposition, in order to reduce cost. In this way, the sputter deposition appears as an interesting alternative to deposit a-Si:H films.

Promising studies present intermediate band (IB) materials as one of the best options for increase the conversion efficiency of solar cells [3]. These materials exhibit sub bandgap photo-response and absorption [4, 5], that allows the absorption of low energy photons, that could increase the conversion efficiency with respect to traditional semiconductors.

Recent researches determined the IB formation by the
R. Garcia-Hernansanz*, E. Garcia-Hemme, J. Olea,

D. Pastor, A. del Prado, I. Martil, G. Gonzalez-Diaz

CEI Campus Moncloa

UCM-UPM

Madrid, Spain

Telf. 913944434, e-mail: * rodrigo.garcia.hernansanz@,fis.ucm.es

\section{F.J. Ferrer}

Centro Nacional de Aceleradores

Universidad de Sevilla-CSIC Sevilla, Spain

implantation of high doses of titanium in silicon followed by a pulsed laser melting treatment [6]. With this procedure, concentrations above the solid solubility for titanium in silicon were achieved. Once the IB material is obtained, the technological challenge is to fabricate a solar cell using a low temperature processes to avoid the degradation of the IB material properties. Because of the reasons exposed previously, HIT solar cell manufactured with sputter deposition, is a promising combination to incorporate an IB material.

The aim of this work is to fabricate and characterize a-Si:H layers deposited by the high pressure sputtering (HPS) technique. When we work in the high pressure range $(\sim 1$ mbar), the atoms that are sputtered from the target with very high energy, collide with the gas atoms and reach the sample thermalized [7]. Therefore, we can deposit a-Si:H layers without sample damage, and form a high quality heterojunction between amorphous and crystalline silicon.

We have studied the different properties of the deposited films in order to achieve the optimal conditions to integrate the films in a HIT solar cell.

\section{EXPERIMENTAL}

Films of a-Si:H have been deposited using a high pressure sputtering system and we have analyzed the composition and optical properties of the films deposited at different RF power and pressure conditions.

The HPS system consists of a deposition chamber, a Huettinger PFG 300 RF supply and a turbomolecular pump supported by a rotatory pump. For better control of the process, we include a shutter, and to allow the best deposition rate we can change the sample-target distance. The target is a 2" crystalline silicon wafer $3 \mathrm{~mm}$ thick, supplied by Kurt J.Lesker Company, with 99,999\% of purity and resistivity below $5 \times 10^{-3} \Omega \mathrm{cm}$. We have worked with high purity $\operatorname{Ar}(6 \times)$ as a process gas, and the residual pressure before start the processes was $4.7 \times 10^{-7}$ mbar.

Previous to film deposition, hydrogen treatment that consists on introducing a $\mathrm{H}(5 \times)$ gas flow in the chamber during 15 minutes without plasma ignition have been used.

NUMANCIA II (Grant No. S-2009/ENE/1477) 
This procedure allows to obtain a hydrogen concentration in the chamber higher than any contamination gas, like $\mathrm{O}$ or $\mathrm{N}$ and, consequently, it favors the formation of $\mathrm{Si}-\mathrm{H}$ bonds in our films. Finalized this process, the sputtering process in pure $\mathrm{Ar}$ atmosphere at 1 mbar have been performed.

In order to control the species present in the plasma, OES (optical emission spectroscopy) technique have been used. Four stellar-Net miniature spectrometers, in the wavelength range from $200 \mathrm{~nm}$ to $1000 \mathrm{~nm}$ have been employed. The realtime control of the plasma emission spectra is an essential tool to know when the start point to deposit the films without contaminant species in the plasma and to prevent vacuum leakages.

The films have been deposited in two substrate types: glass for optical characterization, and both sides polished crystalline silicon for compositional measurements.

FTIR (Fourier Transform Infrared) spectroscopy spectra have been measured using a Nicolet Magna-IR 752 spectrometer, in a wavenumber range from $340 \mathrm{~cm}^{-1}$ to 4000 $\mathrm{cm}^{-1}$. This technique allows to determine the bonding structure of the films, and the hydrogen content from the $\mathrm{Si}-\mathrm{H}$ related absorption bands.

Compositional measurements have been obtained from RBS (Rutherford backscattering spectrometry) for Si, ERDA (elastic recoil detection analysis) for $\mathrm{H}$, and NRA (nuclear reaction analysis) for N. For RBS measurements we use an alpha particles beam of $1.0 \mathrm{MeV}$. In order to avoid the channeling, an inclination of $7^{\circ}$ has been given to the samples and we collected the scatter particles in a detector placed at $165^{\circ}$.To determine the concentration of $\mathrm{N}$ in the samples, the nuclear reaction ${ }^{15} \mathrm{~N}\left(\mathrm{~d}, \alpha_{1}\right){ }^{13} \mathrm{C}$ with deuterons with energy of $1.4 \mathrm{MeV}$ and a detector placed to $150^{\circ}$ as compilation angle have been used. ERDA measurements have been realized using $\mathrm{He}^{2+}$ particles with energy of $3.0 \mathrm{MeV}$. The dispersion angle was $34^{\circ}$, and to detect only particles with this angle, a collimator was placed in front of the detector. To avoid that the scattered $\mathrm{He}^{2+}$ particles arrive to the detector, a $13 \mu \mathrm{m}$ thickness filter of aluminized Mylar was placed too in front of the detector.

To characterize the optical properties of deposited films, transmittance and reflectance measurements have been performed in a Perkin-Elmer lambda 9 spectrophotometer in the range of $300-1700 \mathrm{~nm}$. From these spectra, the absorption coefficient, thickness and the optical band-gap have been determined [8].

\section{RESULTS AND DISCUSSION}

In all sputtering processes the control of the species present in the plasma is important, but in the high pressure range, it is critical as we will see in this discussion.

In Fig. 1 we can observe OES spectra for two different moments in a process. The RF-power was $80 \mathrm{~W}$, the argon flow was $20.1 \mathrm{sccm}$ and the pressure 1 mbar. Spectrum (a) was at the first part to the process, usually is half an hour long, and the shutter cover the sample. In this time, the plasma sputter the first atomic layers from the target, and we must wait to conditioning it to remove the shutter and start the

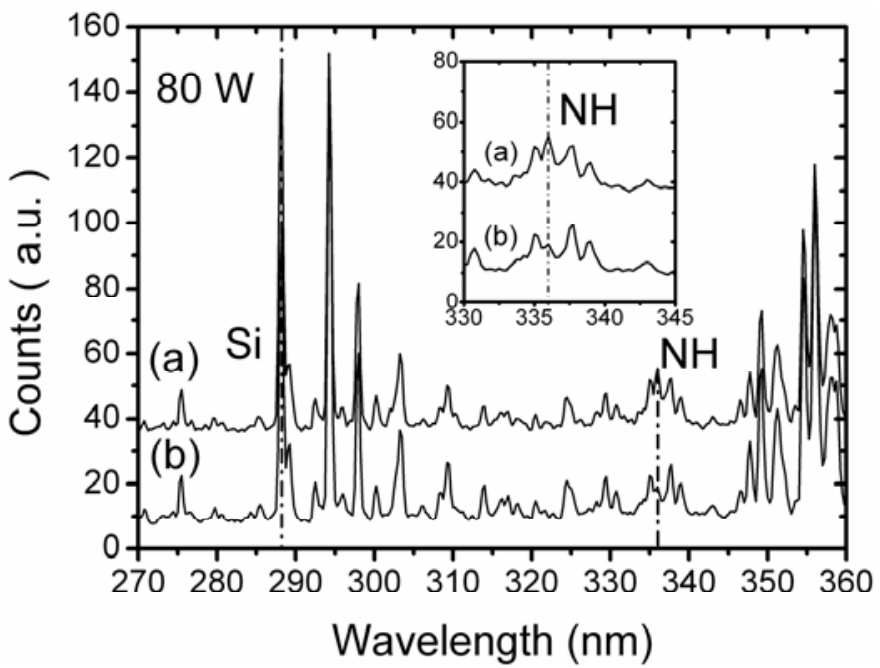

Figure 1. OES spectra for plasma conditioning (a) and deposit process (b) at $\mathrm{RF}$-power of $80 \mathrm{~W}$. We can observe that in (a) the presence of $\mathrm{NH}$ is stronger than in (b). The (a) spectrum has been displaced for clarity.

deposition. During this conditioning, the plasma turn into (b) spectrum gradually. We must focus principally in two plasma emission peaks: one at $336 \mathrm{~nm}$ that corresponds to $\mathrm{NH}$ and the other at $288.1 \mathrm{~nm}$ which is related to silicon [9]. The other peaks present in the figure can be associated to Ar, or are not relevant for this analysis. In the inset of Fig. 1 it is so clear that for spectrum (a), the intensity of the NH peak is above two $\mathrm{Ar}$ peaks situated close it, at $337.75 \mathrm{~nm}$ and at $335 \mathrm{~nm}$, respectively. For spectrum (b) this peak is lower if we compare with the same Ar peaks. This small change, almost negligible, is the difference between the incorporation of about $20 \%$ of $\mathrm{N}$, or less than $5 \%$ in the deposited films. Because of this, the target conditioning is essential. However, the $\mathrm{N}$ present is not always in the $\mathrm{NH}$ form, we have also observed the intensity of the $\mathrm{N}_{2}$ emission peak at $337 \mathrm{~nm}$ [9] above the $\mathrm{NH}$ one, but usually only one of them can be observed. Therefore, we used OES spectrum to monitor the optimal plasma conditions to deposit a-Si:H minimizing the undesirable $\mathrm{N}$ incorporation in the films.

Figure 2 shows the FTIR spectra for films deposited at 1 mbar for different RF power.

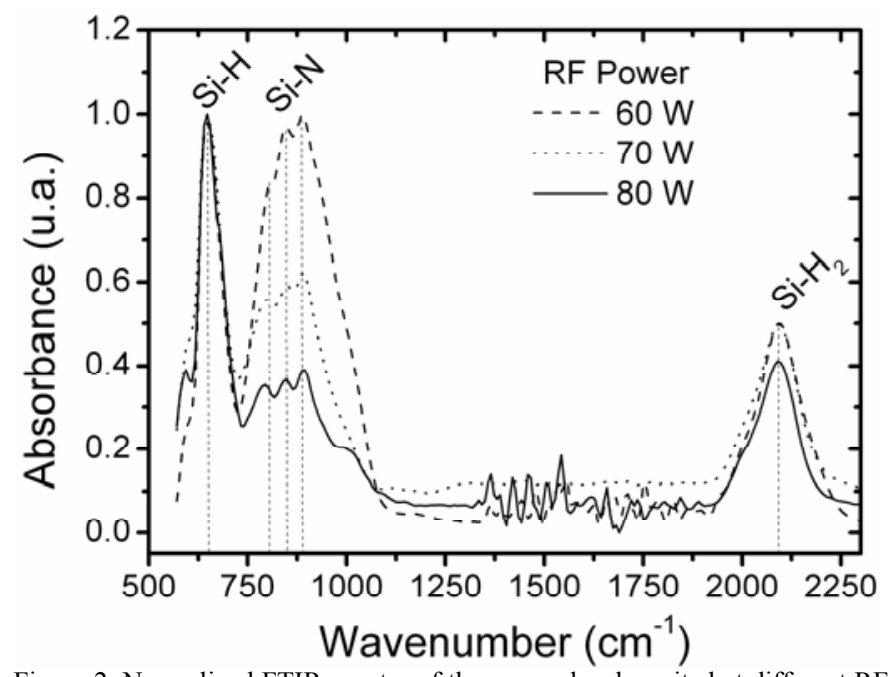

Figure 2. Normalized FTIR spectra of three samples deposited at different RF powers. The Si-N bond concentration is reduced as the RF power is increased. 
For comparative purposes, all the spectra were normalized at the intensity of the FTIR Si-H band located at $640 \mathrm{~cm}^{-1}$. In this figure can be observed two bands at $640 \mathrm{~cm}^{-1}$ and 2090 $\mathrm{cm}^{-1}$ corresponding to the $\mathrm{Si}-\mathrm{H}$ wagging mode [10], and the $\mathrm{Si}-\mathrm{H}_{2}$ stretching mode respectively, as well as three different bands around $850 \mathrm{~cm}^{-1}$. These bands correspond to the $\mathrm{Si}-\mathrm{H}$ bending $\left(802 \mathrm{~cm}^{-1}\right)$, the Si-N stretching $\left(840 \mathrm{~cm}^{-1}\right)$ [11] and the $\mathrm{Si}-\mathrm{H}_{2}$ bending $\left(882 \mathrm{~cm}^{-2}\right)$ [10] modes. The presence of $\mathrm{Si}-\mathrm{N}$ bonds reveals $\mathrm{N}$ incorporation in the deposited layer. To determine the purity of the samples we compare the relative area of the $640 \mathrm{~cm}^{-1} \mathrm{Si}-\mathrm{H}$ and the $840 \mathrm{~cm}^{-1} \mathrm{Si}-\mathrm{N}$ bands. A strong decrease of the relative $\mathrm{Si}-\mathrm{N}$ concentration is observed as the RF power increases.

Fig. 3 shows the concentrations of $\mathrm{Si}, \mathrm{H}$ and $\mathrm{N}$ in the films as a function of RF power. Note that in the process at $40 \mathrm{~W}$, a $30 \%$ of $\mathrm{N}$ was incorporated to the film, while the process at $80 \mathrm{~W}$ only incorporates a $3.1 \%$ of $\mathrm{N}$. According to our results, a RF power above $60 \mathrm{~W}$ is required to deposit a-Si:H with low $\mathrm{N}$ incorporation by HPS when working at pressures around 1 mbar. These measurements are in full agreement with the previously discussed FTIR results. This is because the increase of the RF power improves the extraction of silicon atoms from the target respect to the residual $\mathrm{N}$ in the chamber.

The $\mathrm{NH}$ and $\mathrm{N}_{2}$ emission peaks in OES spectra, are indicative of the presence of contaminant gases in the chamber, and probably its origin is the argon cylinder. In the high pressure range, the Ar cylinder can introduce an important number of impurity atoms in the process, in the same order of magnitude of the silicon atoms extracted from the target. Consequently it is expected that the films deposited at high rate, contain less $\mathrm{N}$, due to the improvement of silicon extraction.

We can use our composition measurements as a reference for calculating the hydrogen content from the FTIR measurements according to the following expression:

$$
[\mathrm{H}]=\mathrm{C} \mathrm{I}
$$

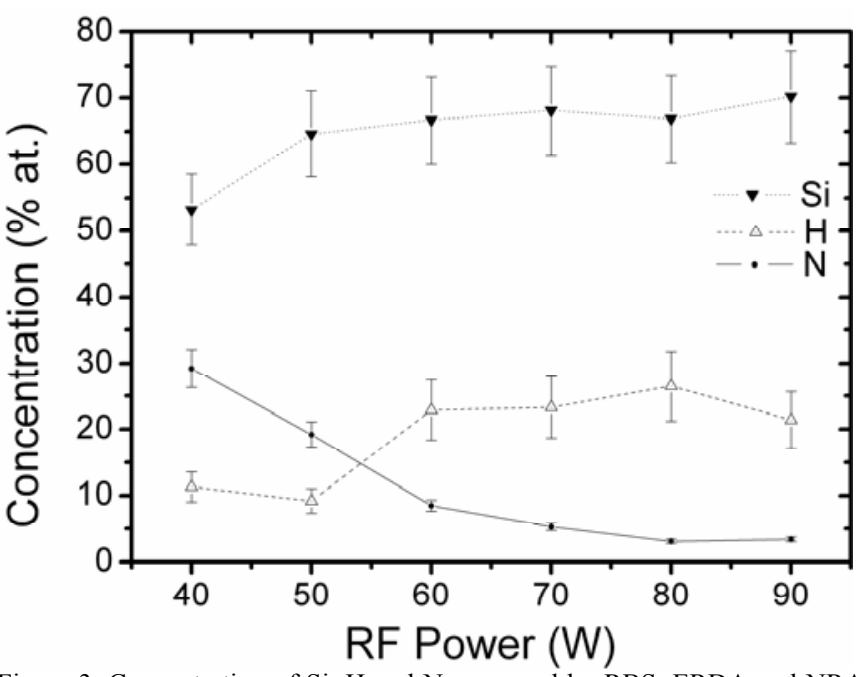

Figure 3. Concentration of $\mathrm{Si}, \mathrm{H}$ and $\mathrm{N}$ measured by RBS, ERDA and NRA, respectively, for films deposited at different RF powers. As we can observe, the minimum RF power to allow a-Si:H with less than $5 \%$ of $\mathrm{N}$ is $80 \mathrm{~W}$.
Table I. Value of $\mathrm{C}$ for differences absorption bands

\begin{tabular}{|c|c|c|c|}
\hline Band & $\begin{array}{c}\text { CVD } \\
\text { Reference }\end{array}$ & $\begin{array}{c}\text { Calculated } \\
\text { value for HPS } \\
\text { layers }\end{array}$ & $\begin{array}{c}\text { Standard } \\
\text { Deviation for } \\
\text { HPS }\end{array}$ \\
\hline $\mathbf{C}_{\mathbf{6 4 0}}$ & $2.1 \times 10^{19} \mathrm{~cm}^{-2}$ & $3.2 \times 10^{19} \mathrm{~cm}^{-2}$ & $0.3 \times 10^{19} \mathrm{~cm}^{-2}$ \\
\hline $\mathbf{C}_{\mathbf{2 0 9 0}}$ & $2.2 \times 10^{20} \mathrm{~cm}^{-2}$ & $1.8 \times 10^{20} \mathrm{~cm}^{-2}$ & $0.2 \times 10^{20} \mathrm{~cm}^{-2}$ \\
\hline
\end{tabular}

Where I is the integrated absorbance of a $\mathrm{Si}-\mathrm{H}$ absorption band in the FTIR spectrum, and C is a constant

$$
I=\int(A / \omega) d \omega
$$

We have calibrated the factor $\mathrm{C}$ from our FTIR and ERDA measurements for both the $640 \mathrm{~cm}^{-1}$ and $2090 \mathrm{~cm}^{-1} \mathrm{Si}-\mathrm{H}$ absorption bands individually. In table 1 we present these results, compared to references for films deposited by CVD [12]. These results were obtained from different samples with $\mathrm{N}$ content ranging from $3.1 \%$ to $30 \%$ and no dependency of the factor $\mathrm{C}$ on the $\mathrm{N}$ content was observed, as we can observe in the standard deviation for HPS value. So it is possible to calculate the $\mathrm{H}$ content from the FTIR spectrum for a-Si:H even when $\mathrm{N}$ is incorporated into the films.

Now we present the results of optical characterization of the films. The band-gap was determined by Tauc form [13], with the extrapolation of the $(\alpha h v)^{1 / 2}$ vs hv curve to $\alpha=0$.

In table 2 we present the values of the gap for different RF power and different deposition times. If we compare these values with other obtained by CVD [14], we observe that the films deposited by HPS have a higher gap. This result is in full agreement with the trend described by Ross and Messier [15], who report an increase of the band-gap value with the process pressure, in parallel with the hydrogen content increase in the deposited films. The hydrogen treatment that we use, and the fact that we work at pressures two orders of magnitude higher, leads us to obtain films with high concentration of hydrogen, which is consistent with these values of the gap.

However, G.D. Cody et al. [14], report a high dependence of the gap with film thickness, reaching values higher than 2 $\mathrm{eV}$ for films with thickness lower than $500 \mathrm{~nm}$. For this low thickness films, they suggest to determine the band-gap with the extrapolation of the $(\alpha / h v)^{1 / 2}$ vs hv curve to $\alpha=0$, to correct the thickness dependence. If we use this correction for our films, we obtain band-gap values close to $1.70 \mathrm{eV}$, which is the reported value for a-Si:H grown by CVD [14].

Table II.Parameters of the films for different RF powers

\begin{tabular}{|c|c|c|c|c|}
\hline $\begin{array}{c}\text { P } \\
(\mathbf{W})\end{array}$ & $\begin{array}{c}\text { Hydrogen } \\
(\mathbf{\%} \text { at. })\end{array}$ & $\begin{array}{c}\text { Thickness } \\
(\mathbf{n m})\end{array}$ & $\begin{array}{c}\text { Gap } \\
(\mathbf{\alpha h v})^{\mathbf{1 / 2}}\end{array}$ & $\begin{array}{c}\text { Gap } \\
(\mathbf{\alpha} / \mathbf{h v})^{\mathbf{1 / 2}}\end{array}$ \\
\hline 60 & 20.8 & 265.5 & 2.07 & 1.68 \\
\hline 70 & 19.3 & 174.2 & 2.10 & 1.66 \\
\hline 80 & 10.6 & 428.7 & 2.00 & 1.79 \\
\hline 90 & 24.5 & 85.5 & 2.15 & 1.68 \\
\hline
\end{tabular}




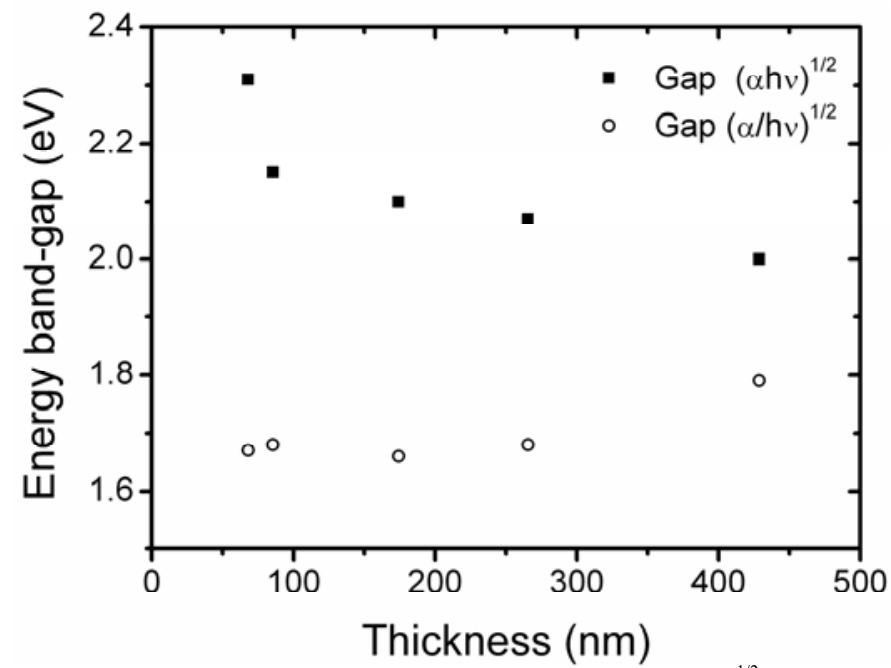

Figure 4. Energy gap vs film thickness. In squares, the $(\alpha h v)^{1 / 2}$ fit, and in circles the $(\alpha / \mathrm{hv})^{1 / 2}$ fit. It is clear how the value of the gap approaches to 1,7 $\mathrm{eV}$ when we fit to $(\alpha / \mathrm{h} v)^{1 / 2}$.

In Fig. 4 we present the optical band-gap calculated by the two methods described before, as a function of film thickness. It is clear that the gap becomes constant at $1.7 \mathrm{eV}$ when we fit to $(\alpha / \mathrm{h} v)^{1 / 2}$ for all films, while a high dependence to the gap with the thickness is observe when we fit to $(\alpha h v)^{1 / 2}$. This result indicates that the fitting proposed by Cody seems appropriate to calculate the band gap for amorphous silicon thin films with thickness below $500 \mathrm{~nm}$.

\section{SUMMARY}

In this work we have optimized the sputtering deposition parameters to improve the quality of the deposited a-Si:H layers with a high-pressure sputtering system.

We have identified the most permanent contamination gases in the plasma by optical emission spectroscopy. This system allows real-time process control and to minimize the $\mathrm{N}$ incorporation into the films by choosing the optimal plasma conditions. We have found a minimum RF-power required to deposit films with low $\mathrm{N}$ incorporation at high pressure.

Besides, by comparing ERDA and FTIR measurements, we have determined the proportionality factors between hydrogen content and the integrated absorbance of the Si-H stretching and wagging modes in hydrogenated amorphous silicon, which are independent of the $\mathrm{N}$ content up to the maximum $30 \% \mathrm{~N}$ content studied in this work.

Also, in this work, we have determined the band gap of the amorphous silicon by two different methods, in order to correct the deviation introduced by the low thickness of the films in Tauc analysis. Fitting to $(\alpha / h v)^{1 / 2}$ we have obtained values for the band-gap around $1.7 \mathrm{eV}$.

This new method to grow a-Si:H could be applied for any devices that need low temperature processing.

\section{ACKNOWLEDGEMENT}

Authors would like to acknowledge C.A.I. de Técnicas Físicas and C.A.I de Espectroscopía of the Universidad Complutense de Madrid for the use of its laboratories and
FTIR measurements. Also we would like to acknowledge Unidad de Energia Solar Fotovoltaica of CIEMAT, for allowing us to measure transmittance and reflectance. This work was partially supported by the Project NUMANCIA II (Grant No. S-2009/ENE/1477) funded by the Comunidad de Madrid.

Research by Eric Garcia-Hemme was partially supported by a PICATA predoctoral fellowship of the Moncloa Campus of International Excellence (UCM-UPM). J. Olea and D. Pastor thanks Professor A. Martí and Professor A. Luque for useful discussions and guidance and acknowledge financial support from the MICINN within the program Juan de la Cierva (JCI-2011-10402 and JCI-2011-11471), under which their research was undertaken.

\section{REFERENCES}

[3] A. Luque and A. Marti, "Increasing the efficiency of ideal solar cells by photon induced transitions at intermediate levels," Physical Review Letters, vol. 78, pp. 5014-5017, Jun 301997.

[4] E. Garcia-Hemme, R. Garcia-Hernansanz, J. Olea, D. Pastor, A. del Prado, I. Martil, et al., "Sub-bandgap spectral photo-response analysis of Ti supersaturated Si," Applied Physics Letters, vol. 101, 5 Nov. 2012.

[5] J. Olea, A. del Prado, D. Pastor, I. Martil, and G. Gonzalez-Diaz, "Sub-bandgap absorption in Ti implanted Si over the Mott limit," Journal of Applied Physics, vol. 109, Jun 12011.

[6] E. Garcia-Hemme, R. Garcia-Hernansanz, J. Olea, D. Pastor, A. del Prado, I. Martil, et al., "Ion implantation and pulsed laser melting processing for the development of an intermediate band material," AIP Conference Proceedings, vol. 1496, 20122012. P. C. Feijoo, A. del Prado, M. Toledano-Luque, E. San Andres, and M. L. Lucia, "Scandium oxide deposited by high-pressure sputtering for memory devices: Physical and interfacial properties," Journal of Applied Physics, vol. 107, Apr 152010.

[8] J. L. Hernandez-Rojas, M. L. Lucia, I. Martil, G. Gonzalez-Diaz, J. Santamaria,and F. Sanchez-Quesada,"Optical analysis of absorbing thin-films application to ternary chalcopyrite semiconductors" Applied Optics, vol. 31, pp. 1606,Apr 11992.

[9] R. W. B. Pearse and A. G. Gaydon, The identification of Molecular Spectra, 4 ed. London, 1976.

[10] A. H. Mahan, L. M. Gedvilas, and J. D. Webb, "Si-H bonding in low hydrogen content amorphous silicon films as probed by infrared spectroscopy and x-ray diffraction," Journal of Applied Physics, vol. 87, Feb 152000.

[11] Y. Rong-Hwei, Y. Tai-Rong, C. Te-Cheng, L. Shih-Yung, and H. Jyh-Wong, "Optoelectronic characteristics of direct-current and alternating-current white thin-film light-emitting diodes based on hydrogenated amorphous silicon nitride film," IEEE Transactions on Electron Devices, vol. 55, April 2008.

[12] A. A. Langford, M. L. Fleet, B. P. Nelson, W. A. Lanford, and N. Maley, "infrared-absorption strength and hydrogen content of hydrogenated amorphous-silicon," Physical Review B, vol. 45, Jun 151992.

[13] J. Tauc, Grigorov.R, and A. Vancu, "optical properties and electronic structure of amorphous germanium," Physica Status Solidi, vol. 15, 19661966.

[14] G. D. Cody, B. G. Brooks, and B. Abeles, "optical-absorption above the optical gap of amorphous-silicon hydride," Solar Energy Materials, vol. 8, 19821982.

[15] R. C. Ross and R. Messier, "microstructure and properties of RFsputtered amorphous hydrogenated silicon films," Journal of Applied Physics, vol. 52, 19811981. 